\title{
Registration of reactions occurring from the emergence of a virus by using an electromagnetic charge effect
}

\author{
Ognyan Ivanov ${ }^{l}{ }^{*}$, Konstantin Simeonov ${ }^{2}$, Petar Todorov ${ }^{1}$, Desislava Antonova ${ }^{l}$, Viktor Pulis ${ }^{1}$ \\ ${ }^{1}$ Georgi Nadjakov Institute of Solid State Physics, Bulgarian Academy of Sciences \\ ${ }^{2}$ Prof. Dr. G. Pavlov National Diagnostic Science-And-Research Veterinary Medical Institute, \\ Pencho Slaveikov 15a Blvd., 1606 Sofia, Bulgaria
}

\begin{abstract}
Early identification of viruses leads to more efficient disease management and control, and is extremely important. A possible new approach for creating virus sensors is the Electromagnetic echo effect (EMEE). An important feature is that the signal from EMEE is highly dependent on the state of the irradiated body. This makes it possible to control ongoing reactions, even if these reactions are invisible to the human eye or other equipment. This article shows the possibility of registering reaction occurring in the presence of an avian coronavirus causing infectious bronchitis, strain Massachusetts. The same methodology can be applied for other types of viruses as well.
\end{abstract}

Keywords: sensor, virus, detection, electromagnetic echo effect

\section{INTRODUCTION}

Throughout history, nothing has claimed more human lives than infectious diseases and in the recent decades global viral outbreaks are becoming more common. The rise in epidemics and the higher risk of pandemics are driven by several global tendencies such as overpopulation, urbanization, increased human mobility, climate change and increased consumption of animal products.

The increasing frequency and magnitude of viral disease outbreaks have resulted in an urgent need for development of fast, reliable and inexpensive methods for virus detection. Early identification of viruses leads to more efficient disease management and control, and is extremely important in order to provide targeted therapy, to decrease mortality, to reduce treatment costs, and last but not least, to identify infected subjects, especially asymptomatic cases, thus preventing the spread of the disease among the population.

There are different methods for viral detection, based mainly on the recognition of viral proteins (antigens) or viral nucleic acids (DNA and RNA, respectively) such as the polymerase chain reaction (PCR) and antigen-specific immunoassays including the enzyme-linked immunosorbent assay (ELISA). These conventional techniques are limited by several factors including requirement for trained personnel, expensive laboratory equipment and reagents, sample pre-processing and time consumption. Therefore, additional scientific approaches have been elaborated, leading to the development of novel biosensor applications, as well as repurposing of already commercialized biosensors.

\footnotetext{
*E-mail: ogi124@yahoo.com, Phone: +3598999 77880
} 
Biosensors are analytical devices designed to detect and/or quantify the presence of a specific biological analyte; they are composed of a biologically active substance (receptor) that is coupled with a transducer responsible for transformation and amplification of the signal, generated by the interaction between the receptor and the analyte. Depending on the type of receptor and transducer, several types of biosensors have been proposed or developed.

The most commonly used bioreceptors are DNA and antibodies. The receptor's role may be also played by enzymes, artificial proteins or peptides, whole cells and cellular membranes, and molecularly imprinted receptors. Although these proteins and membranes can be designed to have high specificity, their production is too expensive for commercial use [1].

According to the transducer implemented, there are electrochemical, optical, piezoelectric, and electronic biosensors. The development of electrochemical biosensors utilise electrical phenomena such as electrostatic potential induced by the binding of molecules, localized surface plasmon resonance (LSPR), plasmonic photothermal (PPT) effect [2], surface acoustic waves (SAW) [3], and whispering-gallery mode in microspherical cavities [4]. Although very promising with respect to sensitivity and selectivity, and suitable for real time viral detection, the reported biosensors based on these effects usually require nanomaterial synthesis, isolation equipment, complex, cost-ineffective and time-consuming fabrication processes and do not allow large-scale production [5].

A possible new approach for creating virus sensors is the Electromagnetic echo effect (EMEE). Its essence is that during interaction of a solid body with an electromagnetic field, the body begins to generate an alternating electrical signal that has a frequency equal to the frequency of the irradiating field. The name reflects the idea that by generating an electromagnetic signal, matter gives a peculiar response to the field with which it interacts [6]. The effect exists in all matter. It can even be measured for fluids by irradiating the fluid-solid boundary.

Our hypothesis is that this is a universal property in the entire electromagnetic range. So far, the effect has been observed from $1 \mathrm{~Hz}$ to $1 \mathrm{GHz}$, infrared, visible and the beginning of the ultraviolet range. No studies have been done in the area between $1 \mathrm{GHz}$ and infrared, and for frequencies higher than the ultraviolet range. In these cases, expensive specialised equipment is needed. The signals measured are in the nano - and micro -volt scale, while the measurement itself is contactless and fast. The measurement is performed with suitable equipment such as selective and phase-sensitive voltmeters between an electrode placed near the irradiated body and the total mass of the system. There are no special requirements for the electrode [7].

An important feature is that the signal from EMEE is highly dependent on the state of the irradiated body. Each solid has a specific signal when interacting with a given electromagnetic field. And if changes occur in the body, the measured signal also changes. This allows for various practical applications [8]. One of them is the ability to control ongoing reactions even if these reactions are invisible to the human eye or other equipment. This has been repeatedly confirmed in our research [9-14]. 


\section{EXPERIMENTS AND DISCUSSIONS}

If we wish to detect the presence of a virus, two approaches are possible. One of them is to irradiate the controlled area with a specific field. As mentioned above, each body has a characteristic signal in this field. If the virus gets in the range of the sensor, it will generate a specific signal. In this approach, special measures must be provided to separate the signal of interest from the signals generated by many other particles, which will also fall on the sensor.

Another approach, which is the subject of this paper, is to monitor the occurrence of a specific reaction that takes place only when the desired virus appears. This can be accomplished by using a surface or a fluid, in which a reaction takes place upon contact with the sought virus. This reaction must be specific and be generated only when there is an interaction with the object of interest, thus changing the EMEE signal accordingly. The idea of such a possibility is discussed in [15].

The change in the measured signal occurs because the reaction causes changes in the layer interacting with the virus, which in turn changes the interface (the boundary surface between the solid and the layer). Any variations in the characteristics of the layer will induce a corresponding change in the interface. The measured signal is generated in this exact area. As a result, any changes in the EMEE signal, corresponding to changes in the layer itself, can be registered. It has been previously shown that even tiny changes in the controlled layer induce measurable variations in the EMEE signal, since the electron properties of the surface are substantially influenced by the boundary interface.

In our experiments, we use the following structure: we irradiate a solid body with a laser beam. We then apply a thin strip layer (about $18 \times 2 \mathrm{~mm}$ in length and width, correspondingly) of virus solution on the surface of the solid. This layer is irradiated with a laser beam at one end, which generates the corresponding signal from the EMEE. A micro droplet of antibodycontaining serum is added at the other end, in order to avoid the danger of accidental disturbance of the laser beam, which will lead to a false signal. As a result, a specific antibodyantigen interaction takes place that changes the measured signal.

The idea is illustrated in Figure 1, where the laser irradiates with modulated light the solid substrate (S), (L) is the studied layer that forms an interface (I) with the substrate. The EMEE signal is measured by the means of an electrode (E) using the appropriate apparatus. 


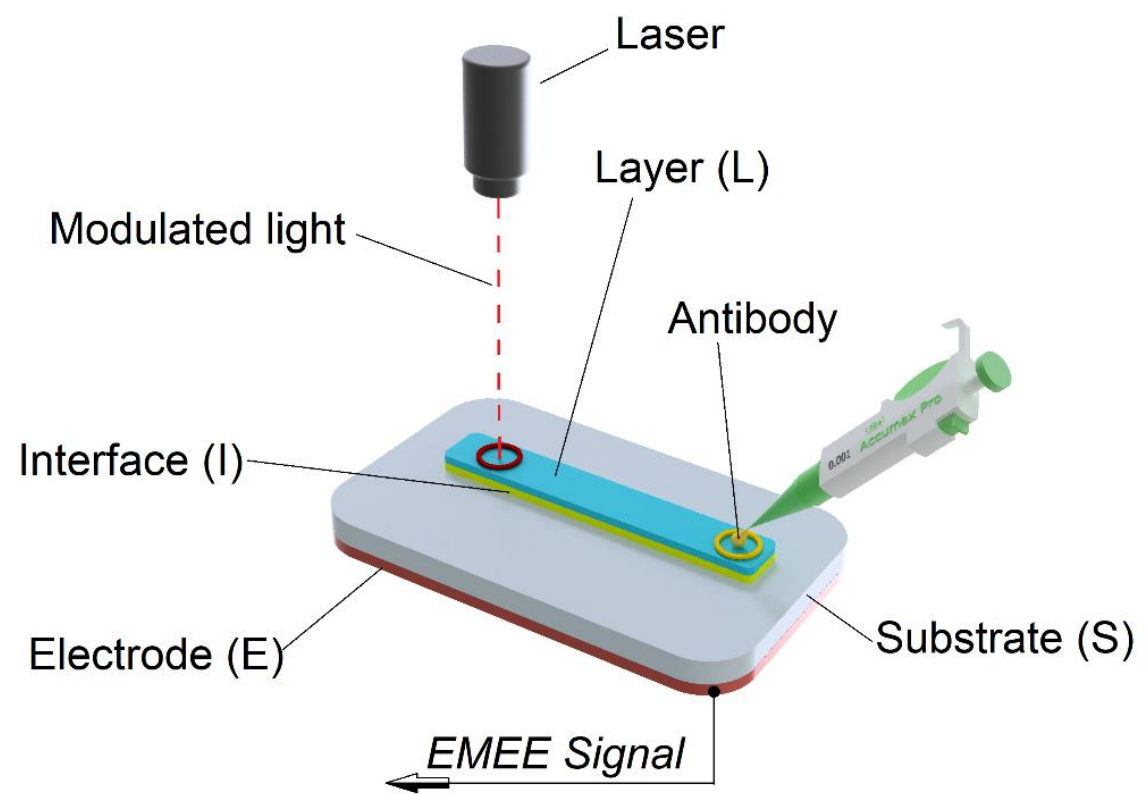

Fig. 1. Possible arrangement of an EMEE - based sensor for viruses: $S$ - solid substrate; $L$ - contact layer; I-solid-liquid interface, generating the signal; E-electrode.

In these experiments, we applied a thin layer with the virus of interest on the solid. After that, a microscopic droplet containing an antibody specific for the virus was added. In case a biosensor for viruses is developed, the experimental setting will be reversed; hence the antiviral antibody should be immobilized on the solid surface. In order to adapt this technology for a mass production and commercial use, a less expensive approach for virus specific interaction should eventually be sought. One such approach is the possibility of development of a reusable solid layer containing artificially synthesized antibodies that are capable of binding antigens with the affinity and selectivity of natural antibodies, but may also be reusable and display superior stability [16]. Of course, the price of the sensor is related also to the purposes it will be used for.

In the experiments conducted until now, the viral species we have used are Chicken anemia virus (CAV) and avian infectious bronchitis virus (IBV), the latter belonging to the viral group of coronaviruses. As a next investigational stage, we intend to commence work with the severe acute respiratory syndrome coronavirus 2 (SARS-CoV-2) - the causative agent for the coronavirus disease 2019 (COVID-19). In this paper, as an example illustrating the feasibility of the method, we present the results obtained from our work with the avian IBV. The biological substances used during the experiments are derived from the following preparations:

1. Commercial vaccine containing the attenuated vaccinal strain Massachusetts $\mathrm{H}-120$ of IBV in a freeze-dried form - CEVAC ${ }^{\circledR}$ BRON 120L

2. IBV enzyme-linked immunosorbent assay (ELISA) kit (IDEXX Laboratories) designed to detect antibodies to IBV in chicken serum. In our experiments, we used IBVimmunoglobulin G (IBV-IgG) negative and IBV-IgG positive control serums from the kit. IBV-IgG is an antibody specific to IBV. 


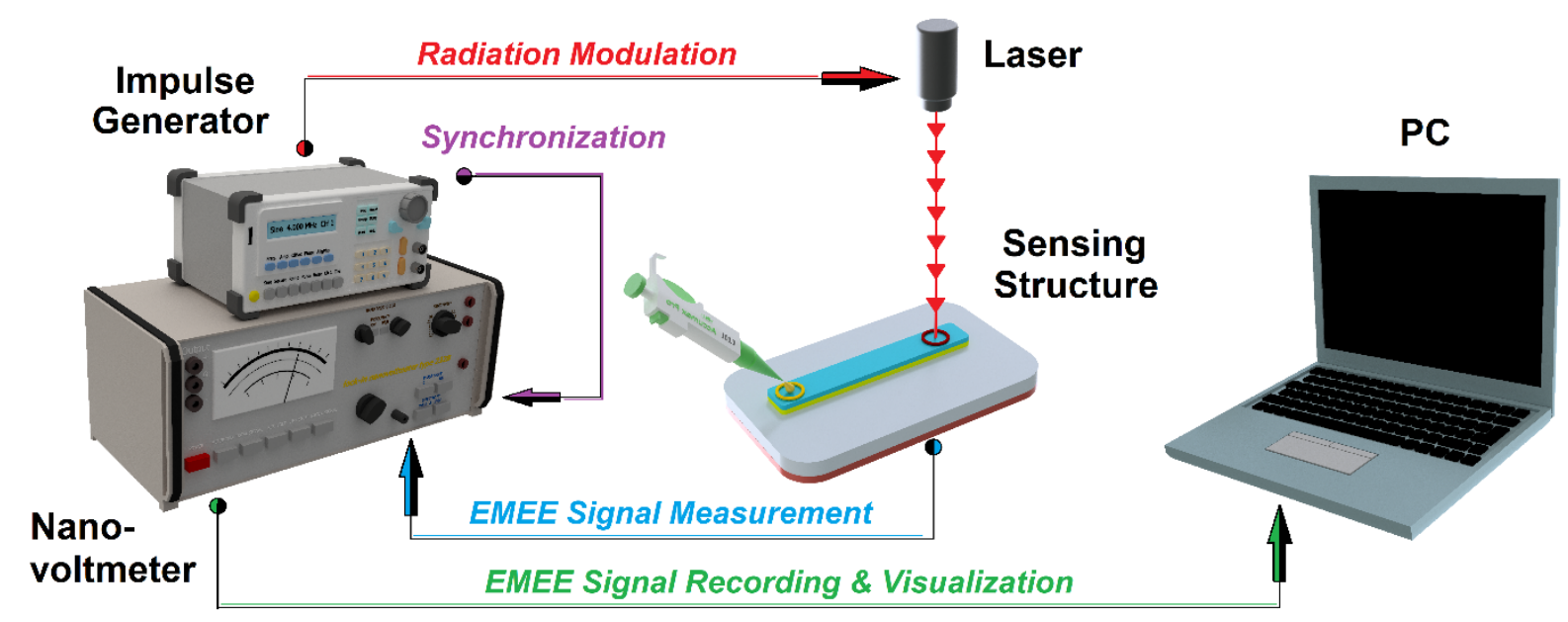

Fig. 2. A block diagram of the experimental system for virus detection

A block diagram of the experimental system is shown in Fig. 2 - an impulse generator modulates the laser radiation falling onto the sensing structure, which generates the EMEE signal of interest that is measured by a nano-voltmeter and transmitted to a computer for recording and visualization. In case a lock-in nano-voltmeter is used for the measurements, the impulse generator sends to it the necessary synchronizing signal. We used mainly semiconductors as substrates and worked with laser radiation in the visible range. The measurements were performed with selective and lock-in nano-voltmeters. The results presented here were obtained with a lock-in nano-voltmeter. A large number of sensory structures were developed that are convenient for conducting this research.

The resulting changes in the EMEE signal for a period of $7 \mathrm{~min}$ after addition of antibodies are demonstrated in Figure 3.

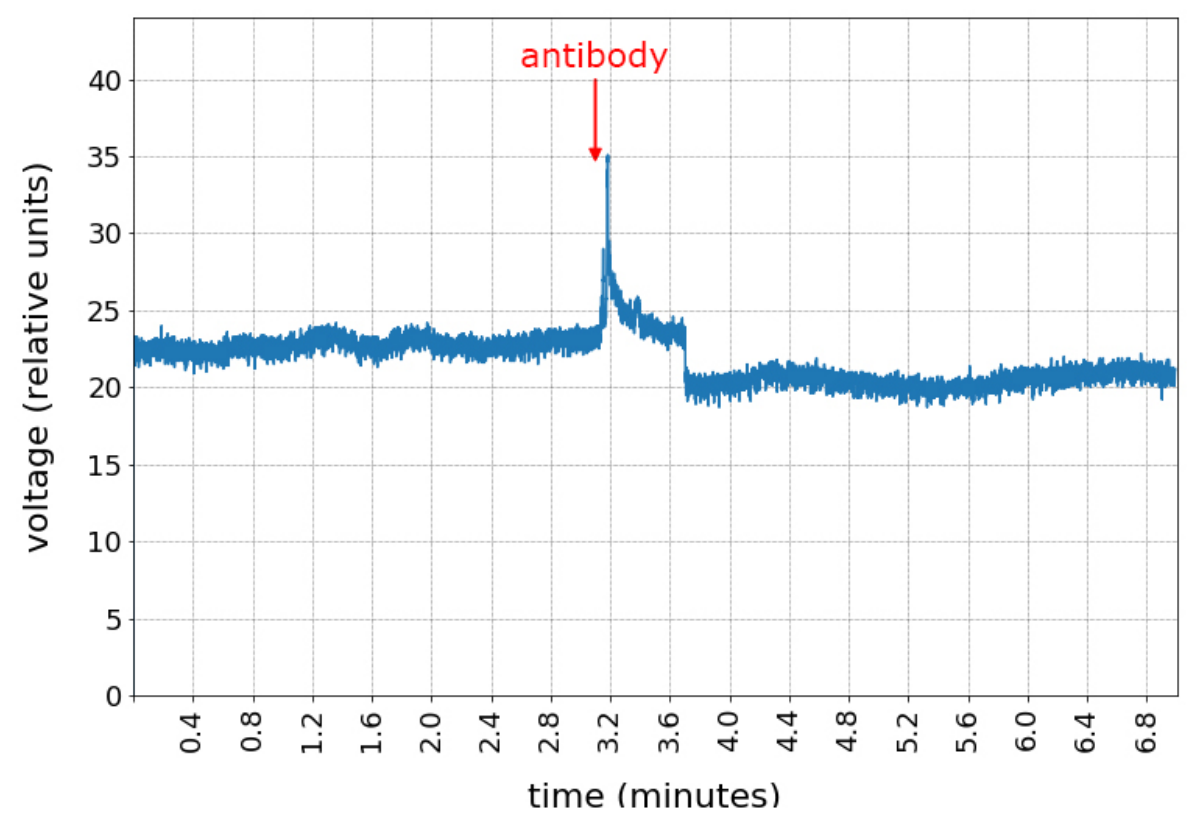

Fig. 3. Recording of the EMEE signal variation for 7 minutes. The layer on the sensor surface contains the avian coronavirus. An arrow indicates the moment when a $10 \mu \mathrm{l}$ sample from the IBV$\mathrm{IgG}$ positive control serum is added. The signal is recorded at an interval of $0.001 \mathrm{~min}$. 
It is clear from the figure that there is a sharp change at the time of addition of the antibodycontaining micro droplet, followed by a relaxation process. There is a potential possibility this relaxation process to be studied under controlled changes in the experimental conditions and appropriate, additional influences. The relaxation process carries information about the ongoing interaction between the virus and the serological reagent.

In order to verify that the change in signal was actually induced by the virus-antibody reaction, experiments were also performed with negative controls. The recording of such an experiment is presented in Fig. 4.

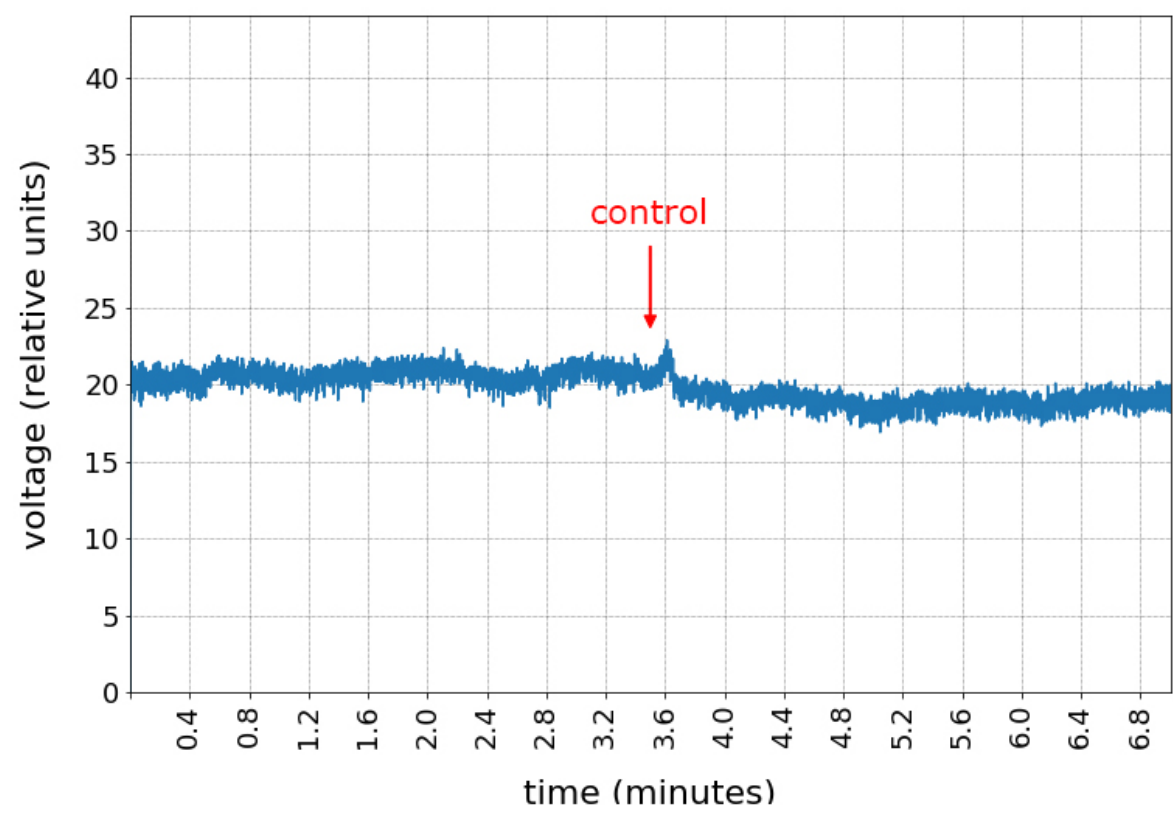

Fig. 4. Recording of the EMEE signal change for $9 \mathrm{~min}$. The layer on the sensor surface contains the avian coronavirus. An arrow indicates the moment when a $10 \mu \mathrm{l}$ sample from the IBV-IgG negative control serum is added. The signal is recorded at an interval of $0.001 \mathrm{~min}$.

The signal along the vertical axis of Figures 3 and 4 is given in relative units because for our purposes it is not necessary to know the exact value of the measured electrical voltage. It is important to note that in the case of real sensors at the moment of emergence of viral particles (when using the current laboratory prototypes we add antibodies) on the sensor, we have a sharp change in the amplitude of the signal. In addition, the amplitude of the signal depends on many parameters and in different experiments, it is a relative value as a function of these parameters.

It can be seen that the measured signal in Fig. 4 differs with almost 12 relative units as compared to that from in Fig. 3. These two graphs demonstrate that the presence of the infectious bronchitis virus can be clearly detected and that it is quite possible to work on the creation of EMEE-based virus sensors. The purpose of this paper is to show this possibility. Figures 3 and 4 show example results. We have conducted multiple similar experiments and the sharp peak in the signal amplitude caused by the virus-antibody interaction was repeatedly observed.

The sensitivity of the proposed method depends on the properties of the interface. Since the surface states are very sensitive to charge redistribution upon illumination, high sensitivity can 
be achieved by selecting appropriate experimental conditions. The measurements are express and contactless, providing the possibility for real-time analysis. Changes in the electrical signal occur without apparent delay. The combination of optical probing of the sample and electrical detection of the generated signal provides convenient, fast and precise measurements. The implementation of the method can be easily realised from technical point of view and without large investments.

It is necessary to select a number of parameters, for the achievement of the desired specific result, such as the type of the structure that generates the signal; the wavelength of the incident radiation; the intensity of the radiation; the parameter of the electrical signal that is to be measured; the material of the irradiated surface; the method for signal registration; the measurement conditions; any additional influences, etc. The desired effect is that a minimal change in the controlled variable to cause maximal changes in the measured electrical signal. After preparation of the set-up for EMEE observation for a specific task, it is crucial that all experimental conditions are kept constant. The choice of specific parameters are technological details and know-how that determine how the system works.

It should be noted that false signals are possible to occur. In the structure described above, the sources of such false signals can be, for example, mechanical disturbances during the addition of the micro droplet to the layer, random reactions occurring between any additional components contained in the virus and antibody solutions, etc. Usually such incorrect signals are easily distinguishable. However, when developing a specific sensor, it is necessary to conduct research and take appropriate measures to ensure the reliability of the readings.

These studies open up the possibility of developing a virus sensor. Its development could proceed in three directions simultaneously - for detection of the virus in the air, onto surfaces and in a fluid from the human body. In the case of air quality control, a constant airflow will be supplied to the sensors, thus providing a continuous control of the space being monitored.

In each case, it is important that the reaction to be detected can occur only in the presence of a particular virus, the appearance of which we would like to control and study. This can be guaranteed by setting up the right conditions and by choosing the appropriate reagents.

\section{CONCLUSION}

In short, the idea of the method is that the measured EMEE signal for fluids is formed by the fluid - solid interface. It is necessary to put the irradiated solid surface in contact with the fluid in which a virus may be present. A reagent capable of generating a specific response for the controlled virus species can be added as well. Since the electron properties of the solid surface are directly influenced by the adjacent fluid layer, it can be expected that optically excited changes in such a system will induce measurable EMEE signals that are generated due to the presence of a specific virus. When all other conditions are constant, the changes caused by the presence of this virus can be detected. This will significantly affect the properties of the illuminated solid - fluid interface and, consequently, the signal from the sensory structure. It can be concluded that the EMEE can be used for development of new techniques for virus presence control. 
The outlined idea presents a great possibility because it combines optical probing of the sample with electrical detection of the generated signal. The main advantage would be the instantaneous results. Such devices will be cheap to produce and compact enough to be used in field work. The proposed approach can be used to detect viruses in the human body, in the air and on solid surfaces.

This method makes it possible both to control the emergence of a virus and to study it. We have in mind that changing the conditions of the experiment can simulate different reactions. By observing the changes in the electrical signals generated by EMEE during these reactions, we will be able to obtain information about the virus.

We will also note that such an approach can be used for express and contactless control of the authenticity of a virus vaccine - whether it has been stored properly and retained its activity. We have carried out successful experiments of this nature. During them, we performed express and non-contact control for changes in the substance of which a series of samples are composed [17], including liquids enclosed in ampoules.

The development of a sensor, based on the electromagnetic echo effect, for the purpose of rapid detection of viruses (SARS-CoV-2 or others, depending on the settings) will strongly support any actions in the fight with diseases constituting public health risk. This new technique has the potential to provide a completely new and robust way to prevent strong negative influences on society in cases of viral outbreaks.

Acknowledgments: This work has been supported by the Bulgarian Ministry of Education and Science under the National Research Programme "Young scientists and postdoctoral students" approved by DCM \# 577 / 17.08.2018. The authors would like to thank Prof. Kostadin Kostadinov from GIS Transfer Center, Adviser to the Minister of the Ministry of Education and Science of Bulgaria, for the support provided in conducting the research.

Author Contributions: Ognyan Ivanov - author of the idea, carried out the measurements; Konstantin Simeonov - provided the biological samples, consultant on biological matters; Petar Todorov - participant in the experiments, formatting of the publication; Desislava Antonova preparation of samples and the texts related to biology; Viktor Pulis - developed the sensory structures.

Conflicts of Interest: The authors declare no conflict of interest.

\section{REFERENCES:}

[1] A. Sadana, Market Size and Economics for Biosensors, Fractal Binding and Dissociation Kinetics for Different Biosensor Applications, 265-299, http://dx.doi.org/10.1016/B978-0-44451945-0.X5000-6 (2005).

[2] G. Qiu et al., Dual-Functional Plasmonic Photothermal Biosensors for Highly Accurate Severe Acute Respiratory Syndrome Coronavirus 2 Detection, ACS Nano, 14 (5), 5268-5277, http://dx.doi.org/10.1021/acsnano.0c02439 (2020). 
[3] K. Länge, F. J. Gruhl, M. Rapp, Surface Acoustic Wave (SAW) biosensors: coupling of sensing layers and measurement, Methods Mol. Biol., 949, 491-505, http://dx.doi.org/10.1007/978-1-62703-134-9_31 (2013).

[4] M. R. Foreman, J. D. Swaim, F. Vollmer, Whispering gallery mode sensors, Advances in Optics and Photonics, 7 (2), 168-240, http://dx.doi.org/10.1364/AOP.7.000168 (2015).

[5] J. Ru Choi, Development of Point-of-Care Biosensors for COVID-19, Front Chem., 8, 517, http://dx.doi.org/10.3389/fchem.2020.00517 (2020).

[6] O. Ivanov, P. Todorov, I. Gultepe, Investigations on the Influence of Chemical Compounds on Fog Microphysical Parameters, Atmosphere, 11 (3), 225, http://dx.doi.org/10.3390/atmos11030225 (2020).

[7] O. Ivanov, M. Kuneva, Quality control methods based on electromagnetic field-matter interactions, InTech, 509-536, http://dx.doi.org/10.5772/15857 (2011).

[8] O. Ivanov, Sensor applications of field-matter interactions. In Encyclopedia of Sensors (American Scientific Publishers, 2006), C. A.Grimes, E. C.Dickey \& M. V. Pishko (editors), 9, 165-197.

[9] O. Ivanov, L. Konstantinov, Investigations of liquids by photo-induced charge effect at solid-liquid interfaces, Sensors and Actuators B, 86 (2-3), 287-289, http://dx.doi.org/10.1016/S0925-4005(02)00215-0 (2002).

[10] O. Ivanov, Level meter for liquids based on the Surface photo-charge effect, Sensors and Actuators B, 75 (3), 210-212, http://dx.doi.org/10.1016/S0925-4005(01)00764-X (2001).

[11] O. Ivanov, S. Radanski, Application of Surface photo charge effect for milk quality control, Journal of Food Science, 74 (7), 79-83, http://dx.doi.org/10.1111/j.17503841.2009.01286.x (2009).

[12] O. Ivanov, S. Karatodorov, J. L. Pérez-Díaz, Novel Electromagnetic Sensor for Contaminations in Fog Based on the Laser-induced Charge Effect, 2017 IEEE SENSORS Proceedings, 1509-1511 (2017).

[13] O. Ivanov, J. L. Perez-Diaz, M. Serkedzhiev, Fog influenced signal generation by Surface photo-charge effect (SPCE), Comptes rendus de l'Académie bulgare des Sciences, 71 (1), 2228, http://dx.doi.org/10.7546/CRABS.2018.01.03 (2018).

[14] O. Ivanov, M. Petrov, H. Naradikian, J. L. Perez-Diaz, Phase transition detection by surface photo charge effect in liquid crystals, Phase Transitions, 91 (5), 449-460, http://dx.doi.org/10.1080/01411594.2018.1431644 (2018).

[15] O. Ivanov, P. Todorov, Zh. Stoyanov, Possibility to create a coronavirus sensor using an optically excited electrical signal, arXiv preprint, arXiv:2010.01965 [physics.app-ph] https://arxiv.org/abs/2010.01965 (2020).

[16] Zh. Zhang et al., Highly stable and reusable imprinted artificial antibody used for in situ detection and disinfection of pathogens, Chemical Science, 6, 2822-2826, http://dx.doi.org/10.1039/C5SC00489F (2015).

[17] O. Ivanov, A. Vaseashta, A method for fast and contactless control of raw materials, Ceramics International, 39 (3), 2903-2907 (2013). 